\title{
Joint value approximation and joint universality for several types of zeta functions
}

by

\author{
TAKASHI NAKAmURA (Nagoya)
}

\section{Introduction}

Definition 1.1. Let $\left\{b_{n}: n \in \mathbb{N}_{0}\right\}, \mathbb{N}_{0}:=\mathbb{N} \cup\{0\}$, be a bounded sequence of complex numbers, and $b: \mathbb{N}_{0} \rightarrow \mathbb{C}$ be a function which satisfies

$$
b(n):=b_{n}+b^{\prime}(n), \quad b^{\prime}(n)=O\left(n^{-\beta}\right), \quad \beta>1 / 2 .
$$

The generalized Barnes zeta function $\zeta(a, b ; s)$ is defined by

$$
\zeta(a, b ; s)=\sum_{n=0}^{\infty} \frac{b(n)}{(n+a)^{s}}
$$

in its region of convergence.

In this article, we assume that the bounded function $b$ satisfies condition (1.1) and $\zeta(a, b ; s)$ can be continued meromorphically to the half-plane $\Re(s)>0$. Needless to say, in the case $a=b(n)=1$, the generalized Barnes zeta function is the Riemann zeta function. When $0<\lambda \leq 1, b_{n}=e^{2 \pi i \lambda n}$, it reduces to the Lerch zeta function $L(\lambda, a, s)$. By modifying the formula [20, p. 85, (10)], we have

$$
\sum_{n_{1}, \ldots, n_{r}=0}^{\infty} \frac{e^{2 \pi i \lambda\left(n_{1}+\cdots+n_{r}\right)}}{\left(n_{1}+\cdots+n_{r}+a\right)^{s}}=\sum_{n=0}^{\infty}\left(\begin{array}{c}
r+n-1 \\
r-1
\end{array}\right) \frac{e^{2 \pi i \lambda n}}{(n+a)^{s}}, \quad \Re(s)>r .
$$

So for $b(n)=e^{2 \pi i \lambda n}\left(\begin{array}{c}r+n-1 \\ r-1\end{array}\right)(n+a)^{1-r}, \zeta(a, b ; s)$ is the (twisted) Barnes $r$-ple zeta function. Our generalization includes the case when $r$ is not an integer.

The distribution of the values of the Riemann zeta function $\zeta(\sigma+i t)$ for fixed $\sigma$ and variable $t>0$ was investigated by H. Bohr. In 1914, he showed the following denseness theorem, in a joint work with R. Courant [4].

2000 Mathematics Subject Classification: Primary 11M06, 11M35, 11M41; Secondary 11M26, $11 \mathrm{~K} 70$.

Key words and phrases: joint value approximation, joint universality, almost periodicity, zeta functions. 
Theorem A (see [14, Theorem 1]). Let $\sigma_{0} \in(1 / 2,1)$. Then the set of values which $\zeta(s)$ takes for $\sigma=\sigma_{0}, t>0$, is everywhere dense in the whole complex plane.

In 1975, S. M. Voronin [22] showed the theorem below, which is now called the universality theorem. To state it, we need some notation. We denote by meas $\{A\}$ the Lebesgue measure of the set $A$, and, for $T>0$, $\nu_{T}^{\tau}\{\ldots\}:=T^{-1}$ meas $\{\tau \in[0, T]: \ldots\}$, where the dots stand for a condition satisfied by $\tau$. Let $D:=\{s \in \mathbb{C}: 1 / 2<\Re(s)<1\}$, and let $K$ and $K_{1}, \ldots, K_{m}$ $(m \geq 2)$ be compact subsets of the strip $D$ with connected complements.

Theorem B (see [10, Theorem 6.5.2]). Let $f$ be a non-vanishing function analytic in the interior of $K$ and continuous on $K$. Then for every $\varepsilon>0$,

$$
\liminf _{T \rightarrow \infty} \nu_{T}^{\tau}\left\{\sup _{s \in K}|\zeta(s+i \tau)-f(s)|<\varepsilon\right\}>0 .
$$

Roughly speaking, this theorem means that any non-vanishing analytic function can be uniformly approximated by the Riemann zeta function $\zeta(s)$. As a generalization of Theorem B, Voronin also proved the next theorem, meaning that a collection of Dirichlet $L$-functions attached to nonequivalent characters uniformly simultaneously approximate non-vanishing analytic functions. In a slightly different form, this was also established independently by Gonek and Bagchi (in unpublished doctoral theses).

Theorem C (see [21, Theorem 1.10]). Let $\chi_{1} \bmod q_{1}, \ldots, \chi_{m} \bmod q_{m}$ be pairwise non-equivalent Dirichlet characters, and $f_{l}$ be a non-vanishing function analytic in the interior of $K_{l}$ and continuous on $K_{l}$ for $1 \leq l \leq m$. Then for every $\varepsilon>0$,

$$
\liminf _{T \rightarrow \infty} \nu_{T}^{\tau}\left\{\sup _{1 \leq l \leq m} \sup _{s \in K_{l}}\left|L\left(s+i \tau, \chi_{l}\right)-f_{l}(s)\right|<\varepsilon\right\}>0 .
$$

We call this property joint universality. Afterwards many mathematicians have considered generalizations of universality (see [21]). For example, the joint universality theorem below was proved by A. Laurinčikas in the case $k_{1}=\cdots=k_{m}$. But it can be easily generalized to the following case.

Theorem D (see [8, Theorem 1]). Let $b_{l}(n):=b_{l n}, n \in \mathbb{N}_{0}, 1 \leq l \leq m$, where $\left\{b_{l n}\right\}$ are periodic sequences of complex numbers with period $k_{l} \in$ $\mathbb{N} \backslash\{1\}$. Define the matrix $B:=\left\{b_{n_{1} n_{2}}\right\}_{1 \leq n_{1} \leq k}^{1 \leq n_{2} \leq m}, m \leq k, k:=\operatorname{lcm}\left(k_{1}, \ldots, k_{m}\right)$, where $\mathrm{lcm}$ is the least common multiple, and suppose $\operatorname{rank}(B)=m$. Let $0<a<1$ be a transcendental number, and $f_{l}$ be functions analytic in the interior of $K_{l}$ and continuous on $K_{l}$. Then for every $\varepsilon>0$,

$$
\liminf _{T \rightarrow \infty} \nu_{T}^{\tau}\left\{\sup _{1 \leq l \leq m} \sup _{s \in K_{l}}\left|\zeta\left(a, b_{l} ; s+i \tau\right)-f_{l}(s)\right|<\varepsilon\right\}>0 .
$$


In this paper, we consider the following property, which is weaker than joint universality, and stronger than joint denseness.

DeFinition 1.2. Joint value approximation (of positive density) for $\zeta\left(a, b_{l} ; s\right)$ is the following property: Let $\sigma_{0} \in(1 / 2,1)$ and $C_{l} \in \mathbb{C}$ for $1 \leq l \leq m$. Then for every $\varepsilon>0$,

$$
\liminf _{T \rightarrow \infty} \nu_{T}^{\tau}\left\{\sup _{1 \leq l \leq m}\left|\zeta\left(a, b_{l} ; \sigma_{0}+i \tau\right)-C_{l}\right|<\varepsilon\right\}>0 .
$$

This concept is also considered by Nagoshi, independently (see [17]).

We can interpret joint value approximation as joint universality in the complex plane. We can also consider joint value approximation as a kind of universality in the case where the compact subset $K$ is a one-point set. These viewpoints are rather important (see Remarks 4.8 and 5.5). We will show that joint value approximation has applications to functional independence (Proposition 5.6), almost periodicity of positive density (Corollaries 5.1 and 5.2), and self-pre-similarity (Corollary 5.4).

This paper is divided into seven sections. In Section 2, we show a limit theorem for the generalized Barnes zeta function $\zeta(a, b ; s)$ (Proposition 2.2).

In Section 3, using that proposition, we obtain a sufficient condition for joint value approximation (Theorem 3.4).

In Section 4, by using the theory of uniform distribution, we show examples of joint value approximation in Examples 4.3, 4.5 and Propositions 4.6 and 4.7. These examples and propositions describe the distribution of values of generalized Barnes zeta functions in the critical strip. Note that these examples show that joint value approximation is easier to prove than joint universality (see Remark 4.4).

In Section 5, by using Propositions 4.6 and 4.7, we show that $\zeta(a, b ; s)$ has a kind of almost periodicity property (see Remark 5.3), almost periodicity of positive density, in the complex plane (Corollaries 5.1 and 5.2). Next, in Corollary 5.4, we obtain self-pre-similarity for $\zeta(s)$ and $L(s, \chi)$ (see Remark 5.5). Finally, by Examples 4.3, 4.5 and Propositions 4.6, 4.7, we obtain the functional independence in Proposition 5.6.

In Section 6, we give a condition enabling one to disprove joint value approximation (Theorem 6.1), and some examples (Examples 6.3 and 6.4). These are also examples of lack of joint universality.

In Section 7, we give conditions implying the lack of joint universality (Theorems 7.2 and 7.3), and obtain an example which has the joint value approximation property but does not satisfy joint universality (Example 7.4). It should be noted that lack of joint universality has already been obtained in [19, Section 6], but together with lack of joint value approximation. 
2. The limit theorem for generalized Barnes zeta function. First, we quote some definitions and theorems from [7] and [11]. Denote by $H(D)$ the space of analytic functions on $D$ equipped with the topology of uniform convergence on compacta, and $H^{m}(D):=H(D) \times \cdots \times H(D)$. We deal with the limit theorem for the analytic function space $H^{m}(D)$, but for the approximation of values, we only need a limit theorem for $\mathbb{C}^{m}$. We remark that all lemmas and propositions below also hold for $\mathbb{C}^{m}$.

Let $\mathfrak{B}(S)$ stand for the class of Borel sets of the space $S$. Define on $\left(H^{m}(D), \mathfrak{B}\left(H^{m}(D)\right)\right)$ the probability measure

$P_{\zeta}^{T}(A):=\nu_{T}^{\tau}\left\{\left(\zeta\left(a, b_{1} ; s+i \tau\right), \ldots, \zeta\left(a, b_{m} ; s+i \tau\right)\right) \in A\right\}, \quad A \in \mathfrak{B}\left(H^{m}(D)\right)$.

What we need is a limit theorem in the sense of weak convergence of probability measures for $P_{\zeta}^{T}$ as $T \rightarrow \infty$, with an explicit form of the limit measure. Denote by $\gamma$ the unit circle on $\mathbb{C}$, and let $\Omega:=\prod_{n=0}^{\infty} \gamma_{n}$, where $\gamma_{n}=\gamma$ for all $n \in \mathbb{N}_{0}$. With the product topology and pointwise multiplication the infinite-dimensional torus $\Omega$ is a compact topological Abelian group. Denoting by $m_{H}$ the Haar probability measure on $(\Omega, \mathfrak{B}(\Omega))$, we obtain a probability space $\left(\Omega, \mathfrak{B}(\Omega), m_{H}\right)$. Let $\omega(n)$ be the projection of $\omega \in \Omega$ to the coordinate space $\gamma_{n}$. Define

$$
\begin{aligned}
\zeta(s, \omega) & :=\left(\zeta\left(a, b_{1} ; s, \omega\right), \ldots, \zeta\left(a, b_{m} ; s, \omega\right)\right), \\
\zeta\left(a, b_{l} ; s, \omega\right) & :=\sum_{n=0}^{\infty} \frac{b_{l}(n) \omega(n)}{(n+a)^{s}}, \quad s \in D, \omega \in \Omega, 1 \leq l \leq m .
\end{aligned}
$$

LEMma 2.1. Let $0<a<1$ be transcendental. Then $\zeta\left(a, b_{l} ; s, \omega\right)$ is an $H^{m}(D)$-valued random element on the probability space $\left(\Omega, \mathfrak{B}(\Omega), m_{H}\right)$.

Proof. By the definition of $\zeta\left(a, b_{l} ; s, \omega\right)$, we have

$$
\sum_{n=0}^{\infty} \frac{\left|b_{l}(n)\right|^{2} \log ^{2} n}{(n+a)^{2 \sigma}}<\infty, \quad \sigma>1 / 2,1 \leq l \leq m .
$$

Hence the assertion follows by modifying the proof of [8, Lemma 2].

Let $P_{\underline{\zeta}}$ stand for the distribution of the random element $\underline{\zeta}(s, \omega)$, i.e.

$$
P_{\underline{\zeta}}(A):=m_{H}(\omega \in \Omega: \underline{\zeta}(s, \omega) \in A), \quad A \in \mathfrak{B}\left(H^{m}(D)\right) .
$$

Proposition 2.2. Suppose $0<a<1$ is a transcendental number and

$$
\zeta\left(a, b_{l} ; s\right)=O\left(t^{\sigma}\right) .
$$

Then the probability measure $P_{\underline{\zeta}}^{T}$ converges weakly to $P_{\underline{\zeta}}$ as $T \rightarrow \infty$.

Proof. For $\sigma>1$, we define

$$
Z_{1}\left(a, b_{l} ; s\right):=\sum_{n=0}^{\infty} \frac{b_{n l}}{(n+a)^{s}}, \quad Z_{2}\left(a, b_{l} ; s\right):=\sum_{n=0}^{\infty} \frac{b_{l}^{\prime}(n)}{(n+a)^{s}} .
$$


By the definitions of $\zeta\left(a, b_{l} ; s\right)$ (see Introduction) and $Z_{2}\left(a, b_{l} ; s\right), Z_{1}\left(a, b_{l} ; s\right)$ can be continued meromorphically to the half-plane $\Re(s)>1 / 2$. From [13, Lemma 2], we have

$$
\lim _{T \rightarrow \infty} \frac{1}{2 T} \int_{-T}^{T}\left|Z_{1}\left(a, b_{l} ; s+i \tau\right)\right|^{2} d \tau=\sum_{n=1}^{\infty} \frac{\left|b_{n l}\right|^{2}}{(n+a)^{2 \sigma}}, \quad \sigma>1 / 2 .
$$

By using $|x+y|^{2} \leq 2\left(|x|^{2}+|y|^{2}\right), x, y \in \mathbb{C}$, and the above formula, we obtain

$$
\int_{0}^{T}\left|\zeta\left(a, b_{l} ; s+i \tau\right)\right|^{2} d \tau=O(T), \quad 1 \leq l \leq m .
$$

Hence the assertion follows by modifying the proof of [8, Theorem 3].

3. A condition for joint value approximation. We quote some wellknown results on weak convergence of probability measures. Suppose $P_{n}$ and $P$ are probability measures on $(S, \mathfrak{B}(S))$ for some metric space $S$.

Lemma 3.1. $P_{n}$ converges weakly to $P$ as $n \rightarrow \infty$ if and only if $\liminf _{n \rightarrow \infty} P_{n}(G) \geq P(G) \quad$ for all open sets $G \in \mathfrak{B}(S)$.

Moreover, we recall that the minimal closed set $S_{P} \subseteq H^{m}(D)$ such that $P\left(S_{P}\right)=1$ is called the support of $P$. The set $S_{P}$ consists of all $f \in H^{m}(D)$ such that $P(V)>0$ for every neighborhood $V$ of $\underline{f}$. The support of the distribution of a random element $X$ is called the support of $X$ and is denoted by $S_{X}$.

Lemma 3.2 ([11, Lemma 2]). Let $\left\{X_{n}\right\}$ be a sequence of independent $H^{m}(D)$-valued random elements, and suppose that the series $\sum_{n=1}^{\infty} X_{n}$ converges almost surely. Then the support of the sum of this series is the closure of the set of all $f \in H^{m}(D)$ which may be written as a convergent series $\underline{f}:=\sum_{n=1}^{\infty} \underline{f}_{n}, \underline{f}_{n}^{-} \in S_{X_{n}}$.

The following lemma plays an important role in the theory of joint value approximation.

Lemma 3.3 ([7, Theorem 6.1.16]). Let $\left\{\underline{x}_{n}\right\}$ be a sequence in a Hilbert space $X$ satisfying the following conditions:

(a) $\sum_{n=0}^{\infty}\left\|\underline{x}_{n}\right\|^{2}<\infty$;

(b) $\sum_{n=0}^{\infty}\left|\left\langle\underline{x}_{n}, \underline{x}\right\rangle\right|=\infty$ for all $0 \neq \underline{x} \in X$.

Then the set of all convergent series $\sum_{n=0}^{\infty} \alpha_{n} \underline{x}_{n}$ with $\left|\alpha_{n}\right|=1$ for all $n \in \mathbb{N}_{0}$ is dense in $X$.

Let $\underline{x}, \underline{y} \in \mathbb{C}^{m}$. We define the inner product in the Hilbert space $\mathbb{C}^{m}$ by

$$
\langle\underline{x}, \underline{y}\rangle:=x_{1} \bar{y}_{1}+\cdots+x_{m} \bar{y}_{m},
$$


where the bar denotes the complex conjugate. From Lemma 3.3, we obtain the following sufficient condition for joint value approximation. It should be noted that such conditions for joint universality have already been proved (see for example [21, Theorem 12.9]).

Theorem 3.4. Let $\sigma_{0} \in(1 / 2,1)$ and $C_{l} \in \mathbb{C}$ for $1 \leq l \leq m$. Suppose $0<a<1$ is transcendental and $\zeta\left(a, b_{l} ; s\right)$ satisfies (2.1) and

$$
\sum_{n=0}^{\infty}\left|\sum_{l=1}^{m} \frac{\bar{\eta}_{l} b_{l}(n)}{(n+a)^{s}}\right|=\infty \quad \text { for all }\left(\eta_{1}, \ldots, \eta_{m}\right) \neq(0, \ldots, 0) .
$$

Then for every $\varepsilon>0$,

$$
\liminf _{T \rightarrow \infty} \nu_{T}^{\tau}\left\{\sup _{1 \leq l \leq m}\left|\zeta\left(a, b_{l} ; \sigma_{0}+i \tau\right)-C_{l}\right|<\varepsilon\right\}>0 .
$$

Proof. We put $\underline{x}_{n}:=\left(b_{1}(n)(n+a)^{-\sigma_{0}}, \ldots, b_{l}(n)(n+a)^{-\sigma_{0}}\right)$. We can check (a) and (b) of Lemma 3.3 easily. We use the fact that

$$
\sup _{1 \leq l \leq m}\left|x_{l}-y_{l}\right| \leq \sqrt{\langle\underline{x}-\underline{y}, \underline{x}-\underline{y}\rangle} \leq m \sup _{1 \leq l \leq m}\left|x_{l}-y_{l}\right|, \quad \underline{x}, \underline{y} \in \mathbb{C}^{m},
$$

which implies the equivalence of the topology defined by $\sup |\cdot|$ and by $\langle\cdot, \cdot\rangle$. Hence the support of the measure $P_{\underline{\zeta}}$ is the whole of $\mathbb{C}^{m}$ by Lemmas 3.2 and 3.3. Denote by $G$ the set of all $\underline{g}:=\left(g_{1}, \ldots, g_{m}\right) \in \mathbb{C}^{m}$ such that

$$
\sup _{1 \leq l \leq m}\left|g_{l}-C_{l}\right|<\varepsilon
$$

Obviously, $G$ is an open set on $\mathbb{C}^{m}$, and as $S_{P_{\underline{\zeta}}}=\mathbb{C}^{m}$, it follows that $\left(C_{1}, \ldots, C_{m}\right) \in S_{P_{\underline{\zeta}}}$. The weak convergence of the measures $P_{\underline{\zeta}}^{T}$ and Lemma 3.1 imply (3.2), since $S_{P_{\zeta}}$ consists of all $\underline{g} \in \mathbb{C}^{m}$ for which every neighborhood $G$ of $\underline{g}$ satisfies $P_{\underline{\zeta}}(\bar{G})>0$.

Remark 3.5. The general Dirichlet series $L\left(b_{l}, \lambda ; s\right)$ is defined by

$$
L\left(b_{l}, \lambda ; s\right):=\sum_{n=1}^{\infty} b_{l}(n) e^{-\lambda_{n} s},
$$

where $\lambda_{n} \in \mathbb{R}, 0<\lambda_{1}<\lambda_{2}<\cdots, \lim _{n \rightarrow \infty} \lambda_{n}=\infty$ (see for example [9]). It is well known that the region of absolute convergence of (3.4) is a half-plane. Assume (3.4) converges absolutely for $\sigma_{l}>\sigma_{a_{l}}$. It is proved in [9, Theorem 1] that if $\lambda_{n}$ 's are linearly independent over the field of rational numbers, and satisfy the conditions $[9,(2),(3),(6)]$, then we have the limit theorem. In this case, we can also prove joint value approximation by modifying the method in this section.

Similarly, if a certain type of Dirichlet series has an Euler product and satisfies suitable conditions, we can also deduce joint value approximation (see for example [21, Theorem 4.3]). 
4. Examples of joint value approximation. In this section, we show examples of joint value approximation (see Examples 4.3 and 4.5, and Propositions 4.6 and 4.7 ). First, we quote a definition and a lemma on uniform distribution.

For a real number $x$, let $[x]$ denote the integer part of $x$, and $\{x\}_{\mathrm{f}}:=$ $x-[x]$ the fractional part of $x$. Let $\left\{x_{n}\right\}$ be a given sequence of real numbers. For a positive integer $N$ and a subset $E$ of $[0,1)$, define the counting function $A\left(E ; N ;\left\{x_{n}\right\}\right)$ as the number of terms $x_{n}, 1 \leq n \leq N$, for which $\left\{x_{n}\right\}_{\mathrm{f}} \in E$.

Definition 4.1. The sequence $\left\{x_{n}\right\}$ of real numbers is said to be $u n i$ formly distributed mod 1 if for every pair $a, b$ of real numbers with $0 \leq a \leq$ $b \leq 1$ we have

$$
\lim _{N \rightarrow \infty} \frac{A\left([a, b) ; N ;\left\{x_{n}\right\}\right)}{N}=b-a .
$$

Lemma 4.2 ([6, Corollary 2.4.2]). Any sequence that is dense in $(0,1)$ can be rearranged to a sequence uniformly distributed mod 1 .

After these preparations, we obtain the following examples.

EXAMPLE 4.3. Joint value approximation holds for $\left\{\zeta\left(a, b_{l} ; s\right)\right\}_{l=1,2}$ when $0<a<1$ is transcendental, $b_{n 1}:=e^{2 \pi i \lambda n}, b_{n 2}:=e^{2 \pi i(\lambda+\theta) n}, 0<\lambda \leq 1$ and $\theta \in \mathbb{R} \backslash \mathbb{Q}$.

Proof. In the case $\eta_{1} \eta_{2}=0$, we can check (3.1) immediately. Now we assume $\eta_{1} \eta_{2} \neq 0$ and put $r e^{2 \pi i \xi}:=-\bar{\eta}_{1} / \bar{\eta}_{2}$. For all $r, \xi \in \mathbb{R}$, we have

$$
\#\left\{n \in \mathbb{N}_{0}: 0 \leq n \leq N,\left|r e^{2 \pi i \xi}-e^{2 \pi i \theta n}\right| \geq 2^{-1 / 2}\right\} \geq 3 N / 5
$$

for sufficiently large $N$. This is proved as follows. If a natural number $n$ satisfies the condition

$$
\pi / 4 \leq \min _{j \in \mathbb{Z}}|2 \pi \xi-2 \pi \theta n+2 \pi j| \leq 7 \pi / 4,
$$

then it also satisfies $\left|r e^{2 \pi i \xi}-e^{2 \pi i \theta n}\right| \geq 2^{-1 / 2}$. Since $\{\theta n\}$ is uniformly distributed $\bmod 1$ because $\theta \in \mathbb{R} \backslash \mathbb{Q}$ (see for example [6, Example 2.1]), we obtain (4.1). Hence for all $\left(\eta_{1}, \eta_{2}\right) \neq(0,0) \in \mathbb{C}^{2}$, we have

$$
\begin{aligned}
\sum_{n=1}^{N}\left|\sum_{l=1}^{2} \frac{\bar{\eta}_{l} b_{l}(n)}{(n+a)^{\sigma_{0}}}\right| & \geq \frac{\left|\eta_{2}\right|}{\sqrt{2}} \sum_{n=3 N / 5}^{N} \frac{1}{(n+a)^{\sigma_{0}}}-\sum_{n=1}^{N} \frac{2 \max _{l=1,2}\left\{\left|\eta_{l}\right|\right\}}{(n+a)^{\sigma_{0}+\min _{l=1,2}\left\{\beta_{l}\right\}}} \\
& \rightarrow \infty, \quad N \rightarrow \infty .
\end{aligned}
$$

We can check (2.1) by applying the assumption $b_{l}^{\prime}(n)=O\left(n^{-\beta_{l}}\right)$ and [10, Theorems 3.1.2 and 3.1.3]. Then Theorem 3.4 yields the result.

REMARK 4.4. We remark that in the cases (i) $\lambda, \theta \in \mathbb{Q} \backslash \mathbb{Z}$, (ii) $\lambda, \theta \in \overline{\mathbb{Q}}$, and (iii) $\lambda \in \mathbb{R}, \theta \in \mathbb{Q} \backslash \mathbb{Z}$, joint universality has already been proved by Laurinčikas and Matsumoto [11, Theorem 1] (see also [12, Theorem 2]), Nagoshi 
[15, Theorem], and the author [18, Theorem 17], respectively. Clearly, joint value approximation follows from joint universality. However, in the case when $\theta \in \mathbb{Q} \backslash \mathbb{Z}$, we can prove the former directly, as follows. For $\theta:=g / h$ and all $\eta \in \mathbb{C}$, we can easily obtain

$$
\#\left\{n \in \mathbb{N}_{0}: 0 \leq n \leq N,\left|\eta-e^{2 \pi i \theta n}\right| \geq 1\right\} \geq N / h .
$$

Therefore we obtain joint value approximation by modifying the proof of Example 4.3.

The key to Example 4.3 is the fact that $\{\theta n\}, \theta \in \mathbb{R} \backslash \mathbb{Q}$, is uniformly distributed $\bmod 1$. The sequence $\{\theta n+d \log (n+a)\}, d \in \mathbb{R}$, is also uniformly distributed (see for example [6, Theorem 3.9]). Hence we obtain the following example.

EXAMPLE 4.5. Joint value approximation holds for $\left\{\zeta\left(a, b_{l} ; s+i d_{l}\right)\right\}_{l=1,2}$ in the case when $0<\lambda \leq 1, \theta \in \mathbb{R} \backslash \mathbb{Q}, b_{n 1}:=e^{2 \pi i \lambda n}, b_{n 2}:=e^{2 \pi i(\lambda+\theta) n}, d_{1}=0$ and $d_{2} \in \mathbb{R}$.

We can also obtain examples of joint value approximation following from the denseness of a sequence.

Proposition 4.6. Suppose $0<a<1$ is transcendental, $0<\lambda \leq 1$, $b_{n 1}=b_{n 2}:=e^{2 \pi i \lambda n}, d_{1}=0$, and $0 \neq d_{2}:=d \in \mathbb{R}$. Then for any $\varepsilon>0$,

$$
\liminf _{T \rightarrow \infty} \nu_{T}^{\tau}\left\{\sup _{l=1,2}\left|\zeta\left(a, b_{l} ; \sigma_{0}+i d_{l}+i \tau\right)-C_{l}\right|<\varepsilon\right\}>0 .
$$

Proof. Some rearrangement of the sequence $\{d \log (n+a)\}, d \neq 0$, is uniformly distributed mod 1 by Lemma 4.2 and the fact that $\{d \log (n+a)\}_{\mathrm{f}}$ is dense in $[0,1)$. Hence we find that for all $\left(\eta_{1}, \eta_{2}\right) \neq(0,0) \in \mathbb{C}^{2}$, a rearrangement of the series

$$
\sum_{n=1}^{\infty}\left|\sum_{l=1}^{2} \frac{\bar{\eta}_{l} e^{2 \pi i \lambda n}}{(n+a)^{\sigma+i d_{l}}}\right|
$$

is divergent by using $(n+a)^{i d}=\exp (i d \log (n+a))$ and modifying the proof of Example 4.3. We can easily show that if a rearrangement of positive terms is divergent, the original series is also divergent. This yields the assertion.

By modifying the proof of the above proposition, we obtain the following result. We remark that $C_{1}, C_{2} \in \mathbb{C}$ do not have to be non-zero.

Proposition 4.7. Suppose $\sigma_{0} \in(1 / 2,1), d_{1}=0$, and $0 \neq d_{2} \in \mathbb{R}$. Then for any $\varepsilon>0$,

$$
\liminf _{T \rightarrow \infty} \nu_{T}^{\tau}\left\{\sup _{l=1,2}\left|L\left(\sigma_{0}+i d_{l}+i \tau, \chi\right)-C_{l}\right|<\varepsilon\right\}>0 .
$$


Proof. We only have to prove this result for the Riemann zeta function. Since $\zeta(s)$ has the Euler product expression, we have

$$
\log \zeta(s)=-\sum_{n=1}^{\infty} \log \left(1-p_{n}^{-\sigma-i t}\right), \quad \sigma>1,
$$

where $p_{n}$ denotes the $n$th prime number. Next we define a $\mathbb{C}^{2}$-valued random element $\log \underline{\zeta}(\sigma, \omega)$ by

$$
\log \underline{\zeta}(\sigma, \omega):=\sum_{n=1}^{\infty}\left(\log \left(1-\frac{\omega\left(p_{n}\right)}{p_{n}^{\sigma}}\right), \log \left(1-\frac{\omega\left(p_{n}\right)}{p_{n}^{\sigma+i d}}\right)\right), \quad\left|\omega\left(p_{n}\right)\right|=1 .
$$

By using the prime number theorem and the denseness of the sequence $\left\{\log p_{n}\right\}_{\mathrm{f}}$ in $(0,1)$, and modifying the proof of Proposition 4.6, we obtain

$$
\sum_{n=1}^{\infty}\left|\sum_{l=1}^{2} \eta_{l} \log \left(1-\frac{\exp \left(-i d_{l} \log p_{n}\right)}{p_{n}^{\sigma} 0}\right)\right|=\infty \quad \text { for all }\left(\eta_{1}, \eta_{2}\right) \neq(0,0) .
$$

Hence by the Taylor expansion of $\log (1-z)$,

$$
\omega \log (1-z) \sim \omega z \sim \log (1-\omega z), \quad|\omega|=1,|z|<1
$$

(see also the proof of [7, Lemma 5.4]), and Lemmas 3.2 and 3.3, the support of $\log \underline{\zeta}(\sigma, \omega)$ is $\mathbb{C}^{2}$. The map $h: \mathbb{C}^{2} \rightarrow \mathbb{C}^{2}$ defined by

$$
\left(x_{1}, x_{2}\right) \mapsto\left(\exp x_{1}, \exp x_{2}\right)
$$

is continuous and sends $\log \underline{\zeta}(\sigma, \omega)$ to $\underline{\zeta}(\sigma, \omega)$ and $\mathbb{C}^{2}$ to $(\mathbb{C} \backslash\{0\})^{2}$. On the other hand, the support of $\bar{\zeta}(\sigma, \omega)$ is closed. Since the closure of $(\mathbb{C} \backslash\{0\})^{2}$ is $\mathbb{C}^{2}$, the support of $\zeta(\sigma, \omega)$ is also $\mathbb{C}^{2}$. By using the limit theorem for $\zeta(s)$ (see [7, Section 5] or [21, Sections 4 and 12]) and modifying the proof of Theorem 3.4, we obtain the assertion.

REMARK 4.8. Propositions 4.6 and 4.7 nearly coincide with the Shifts Universality Principle [5, p. 311] (see also [21, p. 203]) if $b_{1}(n)=b_{2}(n)$ and the compact set is a one-point set. We can obtain these propositions by using the universality theorem (see for example [16, Corollary 2.3]).

5. Applications of joint value approximation. By putting $b_{1}(n)=$ $b_{2}(n)$ and $C:=C_{1}=C_{2}$ in Proposition 4.6 or 4.7 and using the following triangle inequality:

$$
\begin{aligned}
\left|\zeta\left(a, b ; \sigma_{0}+i d+i \tau\right)-\zeta\left(a, b ; \sigma_{0}+i \tau\right)\right| \\
\quad \leq\left|\zeta\left(a, b ; \sigma_{0}+i d+i \tau\right)-C\right|+\left|\zeta\left(a, b ; \sigma_{0}+i \tau\right)-C\right|,
\end{aligned}
$$

we obtain the following corollary, which means that generalized Barnes zeta functions have the property of almost periodicity of positive density, embodied in (5.1) below. These corollaries are also obtained by putting $K:=\{$ one-point set $\}$ in $[5$, Theorem 4$]$ (see also [21, Theorem 10.6]). 
Corollary 5.1. Suppose $0<a<1$ is transcendental, $\sigma_{0} \in(1 / 2,1)$, $0<\lambda \leq 1, b_{n 1}=b_{n 2}:=e^{2 \pi i \lambda n}$, and $0 \neq d \in \mathbb{R}$. Then for any $\varepsilon>0$,

$$
\liminf _{T \rightarrow \infty} \nu_{T}^{\tau}\left\{\left|\zeta\left(a, b ; \sigma_{0}+i d+i \tau\right)-\zeta\left(a, b ; \sigma_{0}+i \tau\right)\right|<\varepsilon\right\}>0 .
$$

Corollary 5.2. Suppose $\sigma_{0} \in(1 / 2,1)$ and $0 \neq d \in \mathbb{R}$. Then for any $\varepsilon>0$,

$$
\liminf _{T \rightarrow \infty} \nu_{T}^{\tau}\left\{\left|L\left(\sigma_{0}+i d+i \tau, \chi\right)-L\left(\sigma_{0}+i \tau, \chi\right)\right|<\varepsilon\right\}>0 .
$$

REMARK 5.3. An analytic function $f(s)$, defined on some vertical strip $a<\sigma<b$, is called almost periodic in the sense of Bohr (or uniformly almost periodic) if, for any positive $\varepsilon>0$, and any $\alpha, \beta$ with $a<\alpha<\beta<b$, there exists a length $l:=l(f, \alpha, \beta, \varepsilon)>0$ such that every interval $\left(\tau_{1}, \tau_{2}\right)$ of length $l$ contains an almost period of $f$ relative to $\varepsilon$ in the closed strip $\alpha \leq \sigma \leq \beta$, i.e., there exists $d \in\left(\tau_{1}, \tau_{2}\right)$ such that

$$
|f(\sigma+i d+i \tau)-f(\sigma+i \tau)|<\varepsilon, \quad \alpha \leq \sigma \leq \beta, \tau \in \mathbb{R} .
$$

Bohr [3] proved that every Dirichlet series is almost periodic in the sense of Bohr in its half-plane of absolute convergence. Moreover, he showed that if $\chi$ is non-principal, then the Riemann hypothesis for the Dirichlet $L$-function $L(s, \chi)$ is equivalent to the almost periodicity in the sense of Bohr of $L(s, \chi)$ in $\sigma>1 / 2$ (see also [21, Section 8.2]).

Next we consider the property of "self-similarity" which resembles almost periodicity for zeta functions. Considering the case of $m=1$ in Proposition 4.7 and putting $C_{1}=L\left(\sigma_{1}, \chi\right)$, we obtain the following corollary.

Corollary 5.4. Let $\sigma_{0}, \sigma_{1} \in(1 / 2,1)$. Then for any $\varepsilon>0$,

$$
\liminf _{T \rightarrow \infty} \nu_{T}^{\tau}\left\{\left|L\left(\sigma_{0}+i \tau, \chi\right)-L\left(\sigma_{1}, \chi\right)\right|<\varepsilon\right\}>0 .
$$

Remark 5.5. More than 50 years after Bohr's paper [3], Bagchi in his Ph.D. thesis [1] proved that the Riemann hypothesis is true if and only if the Riemann zeta function can be approximated by itself in the sense of universality.

The Riemann hypothesis is true if and only if, for any compact subset $K$ in the strip $D$ with connected complement and for any $\varepsilon>0$,

$$
\liminf _{T \rightarrow \infty} \nu_{T}^{\tau}\left\{\max _{s \in K}|\zeta(s+i \tau)-\zeta(s)|<\varepsilon\right\}>0
$$

(see also [21, Theorem 8.3]). In Bagchi [2, Theorem 3.7], it is shown that the above statement also holds for $L(s, \chi)$ in place of $\zeta(s)$. We call this property self-similarity and the property in Corollary 5.4 with $\sigma_{0}=\sigma_{1}$ self-pre-similarity. We can say that self-pre-similarity is self-similarity on a one-point set. 
Note that self-similarity implies almost periodicity in the sense of Bohr. By modifying the proof of Theorem $\mathrm{D}$ with $m=1$, we can see that the generalized Barnes zeta function $\zeta(a, b ; s)$ has the universality property (see also [10, Theorem 6.1.1]). Applying the universality Theorem D with $f(s)=$ $\zeta(a, b ; s)$, we obtain self-similarity for $\zeta(a, b ; s)$, which also implies almost periodicity in the sense of Bohr.

Recall that both almost periodicity in the sense of Bohr and self-similarity for $L$-functions are equivalent to the (generalized) Riemann hypothesis. The difference between Corollary 5.2 and almost periodicity in the sense of Bohr lies in the difference between positive density and uniformity. The difference between Corollary 5.4 and self-similarity amounts to the difference between the complex plane and a functional space (difference caused by the fact that the compact set $K$ is a one-point set or not). If we could close one of these gaps, we could prove the Riemann hypothesis. Needless to say, this is very difficult.

Finally, as an application of Examples 4.3, 4.5 and Propositions 4.6 and 4.7, we obtain the following joint functional independence result. We can prove it by modifying the proof of, for example, [10, Theorem 7.2.1].

Proposition 5.6. Let $\zeta\left(a, b_{l} ; s\right), l=1,2$, be as in Examples $4.3,4.5$ or Propositions 4.6, 4.7. Suppose $F$ is a continuous function on $\mathbb{C}^{2}$ and

$$
F\left(\zeta\left(a, b_{1} ; s\right), \zeta\left(a, b_{2} ; s\right)\right)=0
$$

for all $s$. Then $F \equiv 0$.

6. Lack of joint value approximation. The following theorem gives a condition implying that joint value approximation fails to hold.

Theorem 6.1. Let $\sigma_{0} \in(1 / 2,1)$. Suppose there exists $\left(\eta_{1}, \ldots, \eta_{m}\right) \neq$ $(0, \ldots, 0)$ such that

$$
\sum_{n=0}^{\infty}\left|\sum_{l=1}^{m} \frac{\bar{\eta}_{l} b_{l}(n)}{(n+a)^{\sigma_{0}}}\right|<\infty .
$$

Then for all $\tau \in \mathbb{R}$, there exist $\varepsilon>0$ and $C_{l} \in \mathbb{C}, 1 \leq l \leq m$, satisfying

$$
\sup _{1 \leq l \leq m}\left|\zeta\left(a, b_{l} ; \sigma_{0}+i \tau\right)-C_{l}\right| \geq \varepsilon \text {. }
$$

Proof. We can assume that $\eta_{1} \neq 0$ and $C_{l}=0$ for $2 \leq l \leq m$. Put $\eta:=\max _{1 \leq l \leq m}\left|\eta_{l}\right|$ and suppose

$$
\sup _{2 \leq l \leq m}\left|\zeta\left(a, b_{l} ; \sigma_{0}+i \tau\right)\right|<M .
$$


For all $\tau$ satisfying the above inequality, we have

$$
\begin{aligned}
& \left|\eta_{1} \zeta\left(a, b_{1} ; \sigma_{0}+i \tau\right)+(m \eta+1) M\right| \\
= & \left|(m \eta+1) M+\sum_{l=1}^{m} \eta_{l} \zeta\left(a, b_{l} ; \sigma_{0}+i \tau\right)-\sum_{l=2}^{m} \eta_{l} \zeta\left(a, b_{l} ; \sigma_{0}+i \tau\right)\right|>\eta M,
\end{aligned}
$$

because of the equality $\left|(n+a)^{-i \tau}\right|=1$ and

$$
\left|\sum_{l=1}^{m} \eta_{l} \zeta\left(a, b_{l} ; \sigma_{0}+i \tau\right)\right| \leq \sum_{n=0}^{\infty}\left|\sum_{l=1}^{m} \frac{\bar{\eta}_{l} b_{l}(n)}{(n+a)^{\sigma_{0}+i \tau}}\right|<M .
$$

This implies the assertion.

REMARK 6.2. The negation of assumption (3.1) is (6.1). In addition, we can see that the condition in this theorem also implies the lack of joint universality (see [19, Section 6]), which means that there exist $\varepsilon>0$ and $C_{l} \in \mathbb{C}, 1 \leq l \leq m$, for which there does not exist $\tau$ satisfying

$$
\sup _{1 \leq l \leq m} \sup _{s \in K_{l}}\left|\zeta\left(a, b_{l} ; s+i \tau\right)-C_{l}\right| \leq \varepsilon .
$$

By using this theorem, we obtain the following examples where joint value approximation fails to hold. The example below should be compared with Theorem D.

ExAmple 6.3. Define $B:=\left\{b_{n_{1}, n_{2}}\right\}_{1 \leq n_{1} \leq k}^{1 \leq n_{2} \leq m}, m \leq k$, where $b_{n l}, n \in$ $\mathbb{N}_{0}$, are periodic sequences of complex numbers with period $k_{l} \in \mathbb{N} \backslash\{1\}$, $k:=\operatorname{lcm}\left(k_{1}, \ldots, k_{m}\right)$, and suppose $\operatorname{rank}(B)<m$. Then condition $(6.1)$ is satisfied.

As a generalization, we have the following example, which contains $[19$, Proposition 6.4].

Example 6.4. Suppose there exists $\left(\eta_{1}, \ldots, \eta_{m}\right) \neq(0, \ldots, 0)$ such that $\sum_{l=1}^{m} \bar{\eta}_{l} b_{n l}=0$ for all but finitely many $n \in \mathbb{N}_{0}$. Then condition (6.1) is satisfied.

7. Lack of joint universality. The next lemma is a generalization of [19, Theorem 5.2] and a kind of counter-proposition to Lemma 3.3. The assumption that $\sum_{n=1}^{\infty} \alpha_{n} \underline{x}_{n}$ is convergent is essential to prove [19, Theorem 5.2 , but we do not need it in the proof below.

Lemma 7.1. Let $\left\{\underline{x}_{n}\right\}$ be a sequence in a pre-Hilbert space $X$ with an inner product $\langle\cdot, \cdot\rangle$ satisfying the following condition.

(a) There exists a non-zero $\underline{x} \in X$ and $M>0$ such that $\sum_{n=1}^{\infty}\left|\left\langle\underline{x}_{n}, \underline{x}\right\rangle\right|$ $<M$.

Then the set of all series $\sum_{n=0}^{\infty} \alpha_{n} \underline{x}_{n}$ with $\left|\alpha_{n}\right|=1$ for all $n \in \mathbb{N}_{0}$ is not dense in $X$. 
Proof. We can assume $\|\underline{x}\|=1$ by replacing $\underline{x}$ with $\underline{x}\|\underline{x}\|^{-1}$. The conclusion follows from the inequality

$$
\left\|3 M \underline{x}-\sum_{n=0}^{\infty} \alpha_{n} \underline{x}_{n}\right\|^{2} \geq 9 M^{2}\|\underline{x}\|^{2}-6 M \sum_{n=0}^{\infty}\left|\left\langle\underline{x}_{n}, \underline{x}\right\rangle\right|>3 M^{2} .
$$

Theorem 7.2. Suppose that for $1 \leq l \leq m$, the Dirichlet series expression of $\zeta\left(a, b_{l} ; s\right)$ is valid in the critical strip $D$, and there exist functions $g_{l} \in H(D)$ and measures $\mu_{l}$ with compact supports $K_{l} \subset D$ such that

$$
\sum_{n=0}^{\infty}\left|\sum_{l=1}^{m} \int_{\mathbb{C}} \frac{b_{l}(n) \bar{g}_{l}(s)}{(n+a)^{s}} d \mu_{l}(s)\right|<\infty .
$$

Then for all $\tau \in \mathbb{R}$, there exist $\varepsilon>0$ and $f_{l} \in H(D)$ satisfying

$$
\sup _{1 \leq l \leq m} \sup _{s \in K_{l}}\left|\zeta\left(a, b_{l} ; s+i \tau\right)-f_{l}(s)\right| \geq \varepsilon .
$$

Proof. Obviously, $\langle\underline{x}, y\rangle:=\sum_{l=1}^{m} \int_{\mathbb{C}} x_{l} \bar{y}_{l} d \mu_{l}, \underline{x}, y \in H^{m}(D)$, is an inner product in the pre-Hilbert space $H^{m}(D)$. Because $\left|(n+a)^{i \tau}\right|=1$ and the Dirichlet series expression of $\zeta\left(a, b_{l} ; s\right)$ is valid in $D$, we have

$$
\left\{\left(\zeta\left(a, b_{1} ; s+i \tau\right), \ldots, \zeta\left(a, b_{m} ; s+i \tau\right)\right): \tau \in \mathbb{R}\right\} \subset\{\text { the set of all series } S\},
$$

where

$$
S:=\sum_{n=0}^{\infty}\left(\frac{\alpha_{n} b_{1}(n)}{(n+a)^{s}}, \ldots, \frac{\alpha_{n} b_{m}(n)}{(n+a)^{s}}\right), \quad\left|\alpha_{n}\right|=1 .
$$

By Lemma 7.1, the set of all series $S$ is not dense in $H^{m}(D)$ with the norm defined by the inner product $\langle\cdot, \cdot\rangle$. Hence neither is the set $\left\{\left(\zeta\left(a, b_{1} ; s+i \tau\right)\right.\right.$, $\left.\left.\ldots, \zeta\left(a, b_{m} ; s+i \tau\right)\right): \tau \in \mathbb{R}\right\}$. For all $\underline{F} \in H^{m}(D)$, we immediately obtain

$$
\sum_{l=1}^{m} \int_{\mathbb{C}}\left|F_{l}(s)\right|^{2} d \mu_{l}(s) \leq \sum_{l=1}^{m} C_{l} \sup _{s \in K_{l}}\left|F_{l}(s)\right|^{2} \leq C \sup _{1 \leq l \leq m} \sup _{s \in K_{l}}\left|F_{l}(s)\right|^{2},
$$

for some positive constants $C$ and $C_{l}$. This implies the conclusion.

When the Dirichlet series expression of $\zeta\left(a, b_{l} ; s\right)$ is not valid in the critical strip $D$, we can also disprove joint universality by using a limit theorem.

TheOREM 7.3. Suppose $a=b_{l}(n)=1$ or $a$ is transcendental and there exist functions $g_{l} \in H(D)$ and measures $\mu_{l}$ with compact supports $K_{l} \subset D$ satisfying (7.1). Then there exist $\varepsilon>0$ and $f_{l} \in H(D)$ satisfying

$$
\limsup _{T \rightarrow \infty} \nu_{T}^{\tau}\left\{\sup _{1 \leq l \leq m} \sup _{s \in K_{l}}\left|\zeta\left(a, b_{l} ; s+i \tau\right)-f_{l}(s)\right| \leq \varepsilon\right\}=0 .
$$

Proof. Let $\underline{F} \in H^{m}(D)$. Denote by $V_{k}, k=1,2$, the set of $\underline{g} \in H^{m}(D)$ such that

$$
\sup _{1 \leq l \leq m} \sup _{s \in K_{l}}\left|g_{l}(s)-F_{l}(s)\right|<k \varepsilon .
$$


We recall that the support $S_{P}$ consists of all $f \in H^{m}(D)$ such that $P(V)>0$ for every neighborhood $V$ of $\underline{f}$. By using Proposition 2.2, and modifying the proof of Theorem 7.2, we find that the support of the random element $\underline{\zeta}(s, \omega):=\left(\zeta\left(a, b_{1} ; s, \omega\right), \ldots, \zeta\left(a, b_{m} ; s, \omega\right)\right)$ is not the whole of $H^{m}(D)$. Hence there exists a set of analytic functions $f_{l}, 1 \leq l \leq m$, and its neighborhood $V_{2}$ satisfying $P_{\underline{\zeta}}\left(V_{2}\right)=0$. Since $\bar{V}_{1} \subset V_{2}$, we have $P_{\underline{\zeta}}\left(\bar{V}_{1}\right)=0$. Let $P_{n}$ and $P$ be probability measures defined on $(S, \mathfrak{B}(S))$. It is well known that $P_{n}$ converges weakly to $P$ as $n \rightarrow \infty$ if and only if $\limsup _{n \rightarrow \infty} P_{n}(C) \leq P(C)$ for all closed sets $C$. The set $\bar{V}_{1}$ is closed, so we obtain

$$
\limsup _{T \rightarrow \infty} \nu_{T}^{\tau}\left\{\sup _{1 \leq l \leq m} \sup _{s \in K_{l}}\left|\zeta\left(a, b_{l} ; s+i \tau\right)-f_{l}(s)\right| \leq \varepsilon\right\} \leq P_{\underline{\zeta}}\left(\bar{V}_{1}\right)=0 .
$$

This proves that joint universality fails to hold. By a slight modification, we can prove the assertion in the case $a=b_{l}(n)=1$, the Riemann zeta function.

By taking $g_{1}(s) \equiv-g_{2}(s) \equiv 1$ and $d \mu_{1}(s)=d \mu_{2}\left(s+i d_{2}\right)$ in Theorem 7.2, we can obtain the next example of lack of joint universality. We remark that when the compact sets are one-point sets in the following example, we showed joint value approximation in Propositions 4.6.

EXAmple 7.4. Suppose $d_{1}=0,0 \neq d_{2} \in \mathbb{R}$, and $\sum_{n=0}^{N} b_{n}, b_{n}:=b_{n 1}=$ $b_{n 2}$, is uniformly bounded. Then for all $\tau \in \mathbb{R}$, there exist $\varepsilon>0$, compact subsets $K_{1}, K_{2} \subset D$, and $f_{1}, f_{2} \in H(D)$ satisfying

$$
\sup _{l=1,2} \sup _{s \in K_{l}}\left|\zeta\left(a, b_{l} ; s+i d_{l}+i \tau\right)-f_{l}(s)\right| \geq \varepsilon .
$$

REMARK 7.5. When $b_{1}(n)=b_{2}(n)$, Example 7.4 can also be proved as follows. Suppose $K_{1}$ and $K_{2}:=\left\{s+i d: s \in K_{1}\right\}$ are compact subsets of $D$, and $K_{1} \cap K_{2}$ is not empty. In this case, the pair of zeta functions $(\zeta(a, b ; s), \zeta(a, b ; s+i d))$ is essentially one-dimensional in $K_{1} \cap K_{2}$ (see Shifts Universality Principle [5, p. 311] or [21, p. 203]) .

Example 7.4 with $b_{1}(n)=b_{2}(n)$ is a counter-example to the conjecture in $\left[21\right.$, Section 12.5] since $\sum_{p} p^{i-1}=O(1)$. We can obtain this estimate from

$$
\begin{aligned}
\sum_{p} p^{i-1} \sim \int_{2}^{\infty} x^{i-1} d\left(\frac{x}{\log x}\right) & =\left[\frac{x^{i}}{\log x}\right]_{2}^{\infty}-(i-1) \int_{2}^{\infty} \frac{x^{i}}{x \log x} d x \\
& =O(1)+(1-i) \int_{\log 2}^{\infty} \frac{e^{i t}}{t} d t=O(1)
\end{aligned}
$$

REMARK 7.6. For the general Dirichlet series (3.4) and a certain type of Dirichlet series which has the Euler product expression, under suitable conditions, we can also disprove joint value approximation by modifying the process in this section. It should be noted that to disprove joint value 
approximation when $\sum_{n=0}^{N} b_{l n l}$ are uniformly bounded, we do not need a limit theorem. This means that to disprove joint universality, we do not use the fact that $\log p$ or $\log (n+a)$, where $a$ is transcendental, are linearly independent over the field of rational numbers.

To end this paper, we give the following chart for the convenience of reader.

\begin{tabular}{|c|c|c|c|}
\hline Property & $\sum$ or $\prod$ & $\mathbb{C}$ & $H(D)$ \\
\hline $\begin{array}{c}\text { Joint uni. } \\
\left\{\zeta\left(a, b_{l} ; s\right)\right\}\end{array}$ & Series & Exist (Thm. D) & Exist (Thm. D) \\
\cline { 2 - 4 } & Product & Exist (Thm. C) & Exist (Thm. C) \\
\hline $\begin{array}{c}\text { Self- } \\
\text { similarity }\end{array}$ & Series & Exist (Rmk. 5.5) & Exist (Rmk. 5.5) \\
\cline { 2 - 4 } & Product & Exist (Cor. 5.4) & R. H. (Rmk. 5.5) \\
\hline $\begin{array}{c}\text { Joint uni. } \\
\left\{\zeta\left(a, b ; s+i d_{l}\right)\right\}\end{array}$ & Series & Exist (Prop. 4.6) & Not exist (Thm. 7.2, 7.3) \\
\cline { 2 - 4 } & Product & Exist (Prop. 4.7) & Not exist (Thm. 7.2, 7.3) \\
\hline
\end{tabular}

Acknowledgments. I thank Professor Kohji Matsumoto and Professor Hirofumi Nagoshi for very useful advice. The author is supported by JSPS Research Fellowship for Young Scientists (JSPS Research Fellow DC2).

\section{References}

[1] B. Bagchi, The statistical behaviour and universality properties of the Riemann zetafunction and other allied Dirichlet series, Ph.D. thesis, Indian Statistical Institute, Calcutta, 1981

[2] - , A joint universality theorem for Dirichlet L-functions, Math. Z. 181 (1982), 319-334.

[3] H. Bohr, Über eine quasi-periodische Eigenschaft Dirichletscher Reihen mit Anwendung auf die Dirichletschen L-Funktionen, Math. Ann. 85 (1922), 115-122.

[4] H. Bohr and R. Courant, Neue Anwendungen der Theorie der Diophantischen Approximationen auf die Riemannsche Zetafunktion, J. Reine Angew. Math. 144 (1914), 249-274.

[5] J. Kaczorowski, A. Laurinčikas and J. Steuding, On the value distribution of shifts of universal Dirichlet series, Monatsh. Math. 147 (2006), 309-317.

[6] L. Kuipers and H. Niederreiter, Uniform Distribution of Sequences, Wiley, New York, 1974.

[7] A. Laurinčikas, Limit Theorems for the Riemann Zeta-Function, Kluwer, 1996.

[8] —, The joint universality for periodic Hurwitz zeta-functions, Analysis 26 (2006), 419-428.

[9] —, Value distribution of general Dirichlet series. VI, Nonlinear Anal. Model. Control 10 (2005), 235-246.

[10] A. Laurinčikas and R. Garunkštis, The Lerch Zeta-Function, Kluwer, 2002.

[11] A. Laurinčikas and K. Matsumoto, The joint universality and the functional independence for Lerch zeta-functions, Nagoya Math. J. 157 (2000), 211-227.

[12] -, - Joint value-distribution theorems on Lerch zeta-function II, Liet. Mat. Rink. 46 (2006), 238-249. 
[13] A. Laurinčikas, W. Schwarz and J. Steuding, Value distribution of general Dirichlet series. III, in: Analytic and Probabilistic Methods in Number Theory (Palanga, 2001), TEV, Vilnius, 2002, 137-156.

[14] K. Matsumoto, Probabilistic value-distribution theory of zeta-functions, Sugaku Expositions 17 (2004), 51-71.

[15] H. Nagoshi, Joint universality of Lerch zeta-functions, uniform distribution and discrepancy, a talk at the International Conference on Probability and Number Theory 2005, Kanazawa.

[16] - On the universality for L-functions attached to Maass forms, Analysis (Munich) 25 (2005), 1-22.

[17] —, A joint universality theorem and functional independence for automorphic Lfunctions, preprint.

[18] T. Nakamura, Applications of inversion formulas to the joint t-universality of Lerch zeta functions, J. Number Theory 123 (2007), 1-9.

[19] —, The existence and non-existence of joint t-universality for Lerch zeta functions, ibid. 125 (2007), 424-441.

[20] H. M. Srivastava and J. Choi, Series Associated with the Zeta and Related Functions, Kluwer, 2001.

[21] J. Steuding, Value-Distribution of L-functions, Lecture Notes in Math. 1877, Springer, 2007.

[22] S. M. Voronin, Theorem on the universality of the Riemann zeta-function, Izv. Akad. Nauk SSSR Ser. Mat. 39 (1975), 475-486 (in Russian); English transl.: Math. USSRIzv. 9 (1975), 443-453.

Graduate School of Mathematics

Nagoya University

Chikusa-ku, Nagoya, 464-8602, Japan

E-mail: m03024z@math.nagoya-u.ac.jp 